\title{
Aceros sinterizados tratados al vapor para la fabricación de componentes de amortiguadores bitubo.
}

\author{
L.E.G. CAMBRONERO, M.CAMPOS, J.M.RUIZ-ROMAN, J.M. RUIZ-PRIETO \\ E.T.S.I.MINAS, Rios Rosas 21, 28003 Madrid
}

\begin{abstract}
En este trabajo se analizan las propiedades de tres hierros aleados sintetizados y tratados al vapor. Los materiales seleccionados son el $\mathrm{Fe}+2 \mathrm{Cu}, \mathrm{Fe}+0.6 \mathrm{C}+0.5 \mathrm{Cu}, \mathrm{y} \mathrm{Fe}+0.45 \mathrm{P}+2.5 \mathrm{Cu}$, obtenidos por mezcla elemental de polvos. Una vez compactados y sintetizados, se sometieron a un tratamiento de recubrimiento característico de los hierros, como es el tratamiento al vapor. En los materiales sintetizados y tratados al vapor, se determinaron su densidad, dureza y resistencia a tracción, junto con su análisis microestructural. Así mismo dado que algunos de los componentes de los amortiguadores bitubo están sometidos a esfuerzos de fricción, se analizaron el comportamiento tribologíco de los materiales obtenidos tanto en estado sintetizado como tratados al vapor.
\end{abstract}

Palabras clave: aceros sinterizados, tratamiento al vapor, desgaste

Steam Treated sintered steels for sock absorbers components

In this work, properties of three sintered and steam treated alloyed irons were analysed. These materials were $\mathrm{Fe}+2 \mathrm{Cu}$, $\mathrm{Fe}+0.6 \mathrm{C}+0.5 \mathrm{Cu}$, and $\mathrm{Fe}+0.45 \mathrm{P}+2.5 \mathrm{Cu}$, which were obtained by powder mixing. Once pressed and sintered, samples were steam treated (surface treatment which is characteristic of sintered irons). Density, hardness and tensile strength were obtained on sintered and steam treated samples, within their microstructure analysis. Also, a tribological study was carried out since some parts of shock absorbers are working under friction.

Key Words: Sintered steels, steam treatment, wear.

\section{INTRODUCCIÓN}

Entre las diversas piezas que constituyen los amortiguadores bitubo, varias son obtenidas mediante la Pulvimetalurgia (1-3). La complejidad de las formas, sus buenas propiedades y el coste han permitido(4) que sea esta tecnología la que permita la obtención de piezas como del pistón-válvula que posteriormente son sometidas a un tratamiento al vapor para mejorar sus propiedades (5-7). Esta ultima es una de las piezas con mayores solicitaciones dado que tiene que soportar esfuerzos alternativos, la presión del aceite y la transmisión de esfuerzo a través del segmento que asegura el cierre del pistón. En el caso de amortiguadores que han de soportar cargas elevadas, este componente es una fundición de hierro. En estos casos, cuando dicho segmento produce un desgaste del su alojamiento en el pistón, se produce un ruido cuando actúa el amortiguador y se pierde eficiencia en la amortiguación. Por ello, en la selección de materiales para la fabricación de estas piezas, se plantea la necesidad de conocer su comportamiento a desgaste. Para ello se han seleccionado tres materiales que cumplen las especificaciones mecánicas de estas piezas, y analizado su comportamiento en un sistema pin-on disk.

\section{PROCEDIMIENTO EXPERIMENTAL}

Como materiales se seleccionaron tres tipos de polvos de hierro de distinta naturaleza (SC100.26 reducidos, y ABC100.26 atomizado) y composicion (SC y ABC hierros puros y PASC45, hierro con $0.45 \% \mathrm{P}$ ) para obtener las aleaciones de base hierro $\mathrm{Fe}(\mathrm{SC})-2 \% \mathrm{Cu}, \mathrm{Fe}(\mathrm{ABC})-0.6 \mathrm{C}-0.5 \mathrm{Cu}$, y $\mathrm{Fe}-0.45 \mathrm{P}$ (PASC)-2.5Cu mediante mezcla elemental de estos polvos de hierro
(SC100.29, ABC100.26 y PASC45) con cobre y grafito. Las distintas mezclas de polvos se compactaron en matriz flotante a presiones entre 450 (SC y PASC) y 550MPa (ABC) y obtener probetas rectangulares y de tracción. La sinterizacion de los mismos se realizo a $1150^{\circ} \mathrm{C}$ en $\mathrm{N} 2-5 \% \mathrm{H} 2$ durante 30 minutos con una velocidad de $5^{\circ} \mathrm{C} / \mathrm{min}$. Su comportamiento durante la sinterizacion se traduce en una contracción, mayor conforme menor sea su densidad en verde. Posteriormente se sometieron al tratamiento al vapor.

Además de la determinación de la densidad, la dureza y la resistencia a tracción, se estudio el comportamiento tribologico en ensayos del tipo pin-on-disk. Estos se realizaron empleando una bola de acero al cromo, con una carga de $10 \mathrm{~N}$, una velocidad de deslizamiento de $0,2 \mathrm{~m} / \mathrm{s}$ durante $500 \mathrm{~m}$ y en el aceite del amortiguador. Como resultado del ensayo se obtiene la evolución del coeficiente de fricción (Figura 1) y una huella de desgaste que depende de la resistencia al mismo del material.

\section{RESULTADOS}

En los materiales sinterizados la mayor compresibilidad de los polvos de hierro $\mathrm{ABC}$, junto con su mayor presión de compactación conducen (Tablas 1 y 2) a la obtención de altas resistencias y densidades (Figura 2A), y con ello bajas rugosidades. Así mismo el carbono, fósforo y cobre activan la sinterización, y aumentan la dureza del hierro por difusión, si bien en el caso del cobre, se observan las aureolas de difusión del cobre en el hierro. Posteriormente después del tratamiento al vapor, se mejora la densidad y la dureza por la formación 
TABLA 1. DENSIDAD DE LOS MATERIALES COMPACTADOS A DISTINTAS PRESIONES.

\begin{tabular}{|c|c|c|c|}
\hline MPa & SC100.26+2Cu & $\begin{array}{c}\text { ABC100.30+ } \\
0.6 C+0.5 \mathrm{Cu}\end{array}$ & PASC45+2.5Cu \\
\hline 450 & $6.45 \mathrm{~g} / \mathrm{cm}^{3}$ & $6.80 \mathrm{~g} / \mathrm{cm}^{3}$ & $6.45 \mathrm{~g} / \mathrm{cm}^{3}$ \\
\hline 550 & $6.70 \mathrm{~g} / \mathrm{cm}^{3}$ & $6.80 \mathrm{~g} / \mathrm{cm}^{3}$ & $6.75 \mathrm{~g} / \mathrm{cm}^{3}$ \\
\hline 650 & $6.90 \mathrm{~g} / \mathrm{cm}^{3}$ & $7.00 \mathrm{~g} / \mathrm{cm}^{3}$ & $7.00 \mathrm{~g} / \mathrm{cm}^{3}$ \\
\hline
\end{tabular}

TABLA 2. PROPIEDADES MECANICAS DE LOS MATERIALES SINTERIZADOS Y TRATADOS AL VAPOR.

\begin{tabular}{|l|c|c|c|c|c|c|}
\hline & \multicolumn{2}{|c|}{ SC100.26+2Cu } & \multicolumn{2}{c|}{$\begin{array}{c}\text { ABC100.30+ } \\
0.6 \mathrm{C}+0.5 \mathrm{Cu}\end{array}$} & \multicolumn{2}{c|}{ PASC45+2.5Cu } \\
\hline & Sinterizado & $\begin{array}{c}\text { Trat. } \\
\text { al } \\
\text { vapor }\end{array}$ & Sinterizado & $\begin{array}{c}\text { Trat. } \\
\text { al } \\
\text { vapor }\end{array}$ & Sinterizado & $\begin{array}{c}\text { Trat. } \\
\text { al } \\
\text { vapor }\end{array}$ \\
\hline $\begin{array}{l}\text { Resistencia } \\
\text { a traccion, MPa }\end{array}$ & 263 & 320 & 365 & 388 & 382 & 370 \\
\hline alargamiento, \% & 6.2 & 1.9 & 6.8 & 3.7 & 5.8 & 2.4 \\
\hline
\end{tabular}

de magnetita principalmente rodeando los poros presentes en el material y el calentamiento que se realiza durante este tratamiento que mejora la difusión de los aleantes. Igualmente la resistencia a tracción aumenta, bajando el alargamiento al tratarse al vapor (Tabla 2). Este tratamiento conduce a una perdida de carbono en la periferia de los materiales altos en carbono $(A B C+0.6 C+0.5 C u)$, que no da lugar a una perdida de dureza dada la elevada reducción de la porosidad y a la presencia de la magnetita.

Los resultados del comportamiento tribologico (Figura 2B), muestran que los materiales sinterizados presentan mayores desgastes junto con elevados coeficientes de fricción que los tratados al vapor. Sin embargo, entre estos materiales tratados al vapor los valores de dureza y densidad no conducen a unas diferencias considerables en su comportamiento tribologico.

\section{CONCLUSIONES}

Los materiales estudiados mejoran sus propiedades después de ser sinterizados con el tratamiento al vapor. Este solo reduce el alargamiento, mejorando las demás propiedades. Entre los materiales estudiados, si se desea un elevado coeficiente de fricción que evite el movimiento del segmento, es el SC-2Cu el material a elegir. Pero si el segmento ha de girar libremente, el ABC-0.6C$0.5 \mathrm{Cu}$ resultaría él mas indicado.

\section{AGRADECIMIENTOS.}

Deseamos agradecer a HOGANAS por el suministro de los polvos de base hierro, y a SINTERMETAL por el tratamiento al vapor de los materiales sinterizados.

\section{BIBLIOGRAFIA}

1. G. Dowson, "Powder Metallurgy. The process and its products". Ed. EPMA, 19-24, (1992).

2. "Competitiveness of sintered components. Guide to technological alternatives". Ed. ASSINTER, 13-15, (1996)

3. E. Mosca, "Powder Metallurgy Design Manual”. Ed. MPIF, 93-95, (1989).

4. P. Causemann, "Schock absorvers: a PM sucess story". MPR 51, 30-32, 1, (1997).

5. P. Beiss, "Steam Treatement of Sintered parts", Word Conference on PM vol.1, 488496, (1990).

6. L.F. PeaseIII, J.P.Collete, D.A Pease, “Mechanical properties of steam blackened PM materials", Modern Developments in PM 21, 275-299, (1988).

7. A. Molinari, L. Angelini, G.Straffelini, S.Deburtoli, "Technical and economic advantages of continuous steam treatement of sintered parts". Word Congress on PM94, vol III, 2193-2196 (1994).

\section{Coeficiente de friccion}

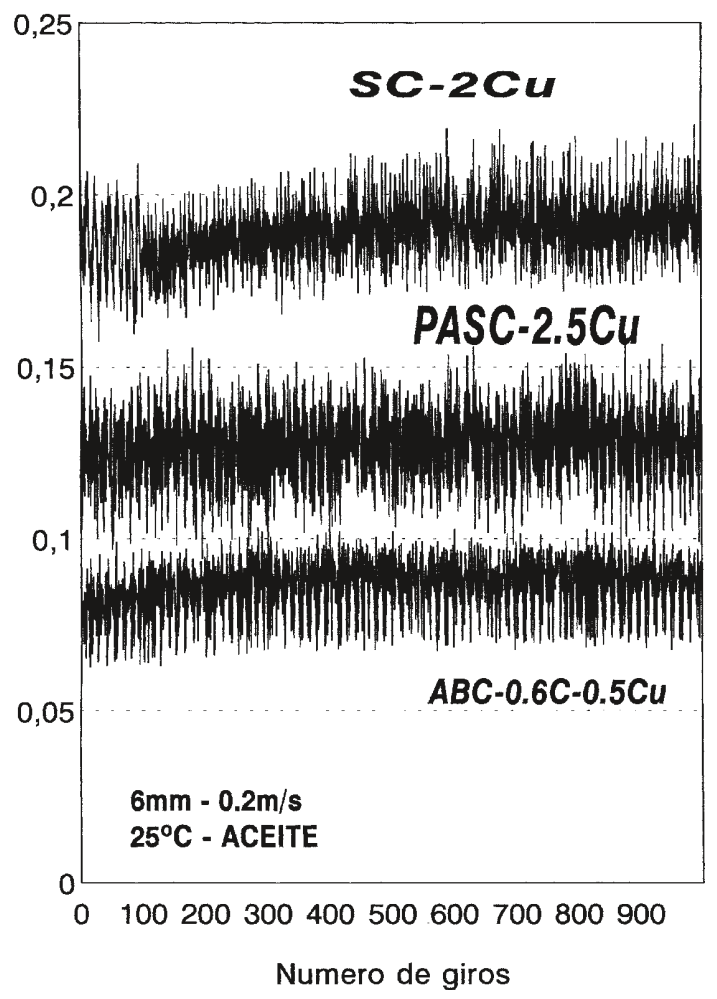

Figura 1. Evolución del coeficiente de fricción en los materiales sinterizados y tratados al vapor durante el ensayo pin-on-disk.

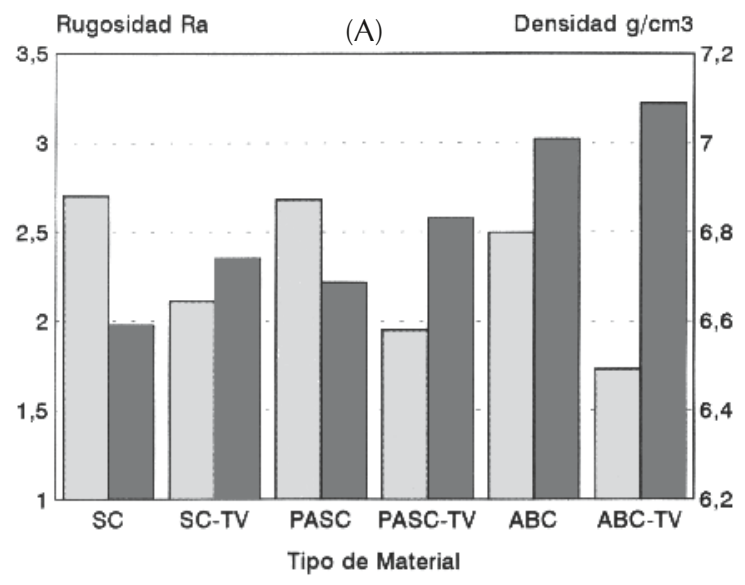

(B)

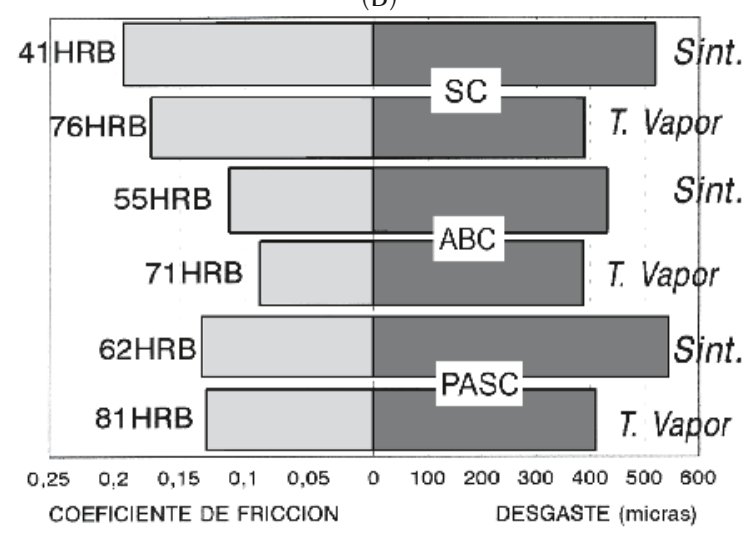

Figura 2. Propiedades físicas (A) y tribolçogicas (B) de los materiales ensayados. 